\title{
Corrections
}

Original Article by Hidetaka Hatori, Tatsuya Zenkoh, Motoo Kobayashi, Yoshihiro Ohtsu, Nobuharu Shigematsu, Hiroyuki Setoi, Motohiro Hino and Hiroshi Handa: FR225659-binding Proteins: Identification as Serine/Threonine Protein Phosphatase PP1 and PP2A Using High-performance Affinity Beads. J Antibiot 57: 456-461 (2004)

should read

\section{FR225659-binding Proteins: Identification as Serine/Threonine Protein Phosphatase PP1 and PP2A Using High-performance Affinity Beads}

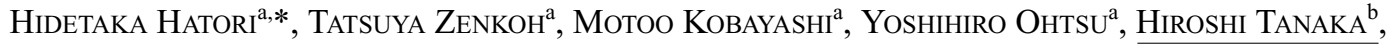 \\ Nobuharu Shigematsu ${ }^{\mathrm{a}}$, Hiroyuki Setoi ${ }^{\mathrm{c}}$, Motohiro Hino ${ }^{\mathrm{d}}$, HARUMA KaWAGuchi $^{\mathrm{g}}$, \\ TAKASHI TAKAHASHI ${ }^{\mathrm{b}}$ and HIROSHI HANDA, \\ ${ }^{a}$ Exploratory Research Laboratories, Fujisawa Pharmaceutical Co., Ltd., \\ 5-2-3 Tokodai, Tsukuba, Ibaraki 300-2698, Japan \\ ${ }^{\mathrm{b}}$ Department of Applied Chemistry, Graduate School of Science and Engineering, Tokyo Institute of Technology, \\ 2-12-1 Ookayama, Meguro, Tokyo 152-8552, Japan \\ ${ }^{\mathrm{c}}$ Medicinal Chemistry Research Laboratories, Fujisawa Pharmaceutical Co., Ltd., \\ 2-1-6 Kashima, Yodogawa, Osaka 532-8514, Japan \\ ${ }^{\mathrm{d}}$ Fermentation Development Laboratories, Fujisawa Pharmaceutical Co., Ltd., \\ 156 Shinkawa, Nishikasugai, Aichi 452-0915, Japan \\ ${ }^{\mathrm{e}}$ Graduate school of Bioscience and Biotechnology and ${ }^{\mathrm{f}}$ Frontier Collaborative Research Center, \\ Tokyo Institute of Technology, \\ Yokohama 226-8503, Japan \\ ${ }^{\mathrm{g}}$ Faculty of Science and Technology, Keio University, \\ Yokohama, Kanagawa 223-8552, Japan \\ (Received for publication January 13, 2004)
}

Consequently, the Contents \& the Author Index should be corrected, respectively.

Original Article by Wenhan Lin, Liya Li, Hongzheng Fu, Isabel Sattler, Xueshi Huang, Susanne Grabley: New Cyclopentenone Derivatives from an Endophytic Streptomyces sp. Isolated from the Mangrove Plant Aegiceras comiculatum. J Antibiot 58: 594-598 (2005)

Author's name should be changed following place:

Liya Li, Wehan Lin, Hongzheng Fu, Isabel Sattler, Xueshi Huang, Susanne Grabley 